\title{
Radial extracorporeal shock wave therapy to support breathing in case of coronavirus disease 2019 (COVID-19): a case report
}

\author{
Peter Stiller ${ }^{l}$ and Christoph Schmitz ${ }^{2}$ \\ ${ }^{I}$ Department of General Medicine, Clinic Lechhausen, Augsburg, Germany \\ ${ }^{2}$ Extracorporeal Shock Wave Research Unit, Chair of Neuroanatomy, Institute of Anatomy, \\ Faculty of Medicine, LMU Munich, Munich, Germany
}

Many patients with Coronavirus disease 2019 (COVID-19) suffer from shortness of breath and severe chest pain. Here we report successful therapy of a patient with diagnosis of COVID-19, severe chest pain and significant shortness of breath, using radial extracorporeal shock wave therapy (rESWT). The latter started seven days after beginning of symptoms and drug therapy without success, and involved daily application of 15.000 to 20.000 radial extracorporeal shock waves over the intercostal muscles as well as the paravertebral muscles of the thoracic and lumbar spine, diaphragm and flanks. Immediately after the first rESWT session the patient experienced significant pain relief and improvement of breathing. Four days later the pain had completely subsided and breathing was largely normalized. This type of noninvasive, non-pharmacologic treatment could help many COVID-19 patients or patients who still suffer from breathing problems weeks after having been infected with SARS-CoV-2, giving them back quality of life.

Correspondence

Dr. Christoph Schmitz MD

Extracorporeal Shock Wave

Research Unit

Chair of Neuroanatomy Institute of Anatomy Faculty of Medicine LMU Munich

Pettenkoferstr. 11 80336 Munich

Germany

Phone: +49 (89) 2180-72620

Fax: +49 (89) 2180-72683

E-mail: chschmitz@med.lmu.de 


\begin{abstract}
Many patients with Coronavirus disease 2019 (COVID-19) suffer from shortness of breath and severe chest pain. Here we report successful therapy of a patient with diagnosis of COVID-19, severe chest pain and significant shortness of breath, using radial extracorporeal shock wave therapy (rESWT). The latter started seven days after beginning of symptoms and drug therapy without success, and involved daily application of 15.000 to 20.000 radial extracorporeal shock waves over the intercostal muscles as well as the paravertebral muscles of the thoracic and lumbar spine, diaphragm and flanks. Immediately after the first rESWT session the patient experienced significant pain relief and improvement of breathing. Four days later the pain had completely subsided and breathing was largely normalized. This type of noninvasive, non-pharmacologic treatment could help many COVID-19 patients or patients who still suffer from breathing problems weeks after having been infected with SARS-CoV-2, giving them back quality of life.
\end{abstract}

\title{
KEYWORDS
}

COVID-19; shortness of breath; chest pain; radial extracorporeal shock wave therapy; rESWT

\section{INTRODUCTION}

Many patients with Coronavirus disease 2019 (COVID-19) suffer from shortness of breath and severe chest pain [1]. The latter can be caused by pleurisy [2], and may substantially contribute to limitations of breathing, potentially further aggravating shortness of breath. Contemporary physical therapy measures may only have limited impact on severe chest pain relief in patients with COVID-19.

\section{CASE REPORT}

This individual case report describes a 13-year-old patient with diagnosis of COVID-19, resulting in significant shortness of breath. Individual case reports are exempt from approval of an Institutional Review Board in Germany. The first author of this case report (P.S.) has been the family doctor of the patient for many years. The patient and the patient's parents (having parental authority) gave informed consent to the treatment and the publication of this individual case report.

On January $10^{\text {th }}, 2021(01 / 10 / 21)$ the otherwise healthy patient developed severe chest pain as well as severe muscle pain in the entire thoracic area and the thoracic spine, lumbar spine and flanks on both sides, which quickly worsened. One day later (i.e., on $01 / 11 / 21$ ) the severity of pain was $7-8$ on a visual analogue scale ranging from zero (no pain) to 10 (maximum, unbearable pain). Furthermore, the patient reported increasing shortness of breath ( 7 on a scale ranging from 0 (breathing freely) to 10 (absolute shortness of breath / suffocation)). A PCR test for severe acute respiratory syndrome coronavirus-2 (SARS-CoV-2) was positive on $01 / 13 / 21$.

Based on suspected diagnosis COVID-19 the following medication was administered to the patient starting $01 / 11 / 21$ : $4 \mathrm{mg}$ of Dexamethasone twice a day, Fluticasone inhalation, $400 \mathrm{mg}$ of Ibuprofen three times a day, $50 \mathrm{mg}$ of Aspirin per day, $600 \mathrm{mg}$ Vitamin $\mathrm{C}$ per day, $20 \mathrm{mg}$ zinc per day, $7000 \mathrm{IE}$ of Vitamin D3 + $200 \mu \mathrm{g}$ Vitamin K2 per week. In addition, the patient received targeted breathing exercises and manual therapeutic treatment. However, despite the high-dose drug therapy and the additional physical therapy, there was no improvement until 01/18/21.

Clinical examination on 01/18/21 revealed the following: patient with clearly subjective and objective dyspnoea; pain in the diaphragm area and the entire lower chest when inhaling deeply; peripheral oxygen saturation $\left(\mathrm{SpO}_{2}\right)=98 \%$; blood pressure $=100 / 65 \mathrm{~mm} \mathrm{Hg}$; heart rate $=96$ beats per minute; minimal crackling sound on basal parts of both lungs, combined with slight wheezing and humming on expiration; significant pain on paravertebral pressure in the thoracic and lumbar spine areas, as well as pain when tapping on both sides; normal urinalysis results. Slightest physical exertion (five steps) caused immediate increase in shortness of breath and rapid exhaustion.

The patient is not known to have any allergic diathesis, asthma or any other pulmonary disease.

Normally the following therapy would have been appropriate at this time: further rest, breathing training, oral and inhalative corticosteroid, inhalation of Salbutamol, and continuation of physical therapy. A pediatric intensive care doctor friend recommended to consider inpatient monitoring.

However, because there was no success with the specific measures that had been performed so far and we no longer wanted to administer corticosteroids to the 13-year-old patient, we decided to completely discontinue medication except for Aspirin and the vitamins. Instead, we started to treat the thorax, intercostal muscles, paravertebral muscles and the diaphragm with radial extracorporeal shock wave therapy (rESWT) using a Swiss DolorClast device (Electro Medical Systems, Nyon, Switzerland). Radial extracorporeal shock waves (rESWs) are single acoustic impulses which have an initial high positive peak pressure of up to 10 megapascals (MPa) that is reached in less than one microsecond $(\mu \mathrm{s})$, followed by a low tensile amplitude of a few $\mu$ s duration that can generate cavitation, and a short life cycle of approximately 10-20 $\mu \mathrm{s}$ [3,4]. Due to these characteristics rESWs fundamentally differ from therapeutic ultrasound. During the last two decades rESWT has emerged as a noninvasive 
management option for tendon and other pathologies of the musculoskeletal system, with only a few unwanted side effects such as temporary skin redness and dyscomfort during treatment $[5,6]$.

Because of potential development of cavitation bubbles [3] treatment over air-filled tissue (lung, gut) is considered a contraindication by the manufacturer of the rESWT device. On the other hand, the first author of this case report (P.S.) has treated a large number of patients suffering from various diseases involving the thorax and diaphragm, including rib fractures and thoracic trauma. In our experience rESWT performed on the thorax and diaphragm can very often lead to rapid pain relief and complete regeneration of breathing, even in cases that were previously refractory to therapy. To our knowledge, treatment of chest pain and breathing problems related to COVID-19 using rESWT has not yet been published.

Radial extracorporeal shock wave therapy started on 01/19/2021 using the following protocol: one rESWT session per day; Swiss DolorClast mobile device (treatments were performed at the patient's home), Blue handpiece, 40-mm applicator, initial air pressure $1.0 \mathrm{bar}$, rESWs applied at $18 \mathrm{~Hz}$, 15.000 to 20.000 rESWs per rESWT session applied over the intercostal muscles from ventral and dorsal as well as the paravertebral muscles of the thoracic and lumbar spine, diaphragm and flanks (Figure 1).

Our plan was to achieve the following therapeutic effects using rESWT: rapid pain relief [5], inhibition of inflammation [7], loosening of mucus in the lungs (mechanical effect of rESWs) and loosening of the diaphragm (also mechanical effect of rESWs) [8].

Already two hours after the first rESWT session there was significant pain relief in the treated areas, as well as about $10 \%$ easier breathing. The air pressure of the rESWT device could rapidly be increased to 1.5 bar in the following days. The patient did not cough during the treatment, indicating that pleura and lung were not irritated by the rESWs.

Four days later (i.e., on 01/23/2021) the pain had completely subsided $(0 / 10)$ and breathing was largely normalized (breathing restriction 0-1/10).

A follow-up examination at a pediatric clinic on $02 / 21 / 2021$ demonstrated no residuals on the lungs, except for a mild restriction of the lung capacity of $7-10 \%$ during exertion. It was decided to continue breathing exercises and performing sport at the discretion of the patient, with the aim to reach full lung capacity during the following four to six weeks.

Another follow-up examination on 02/03/2021 evidenced that the patient had remained pain-free at all areas of the body that were treated with rESWT. Breathing was completely normal at rest. When forcing higher physical exertion there was still no pain but a little restriction of breathing. However, there is progress every week and the patient hopes to be fully rehabilitated in a few weeks.

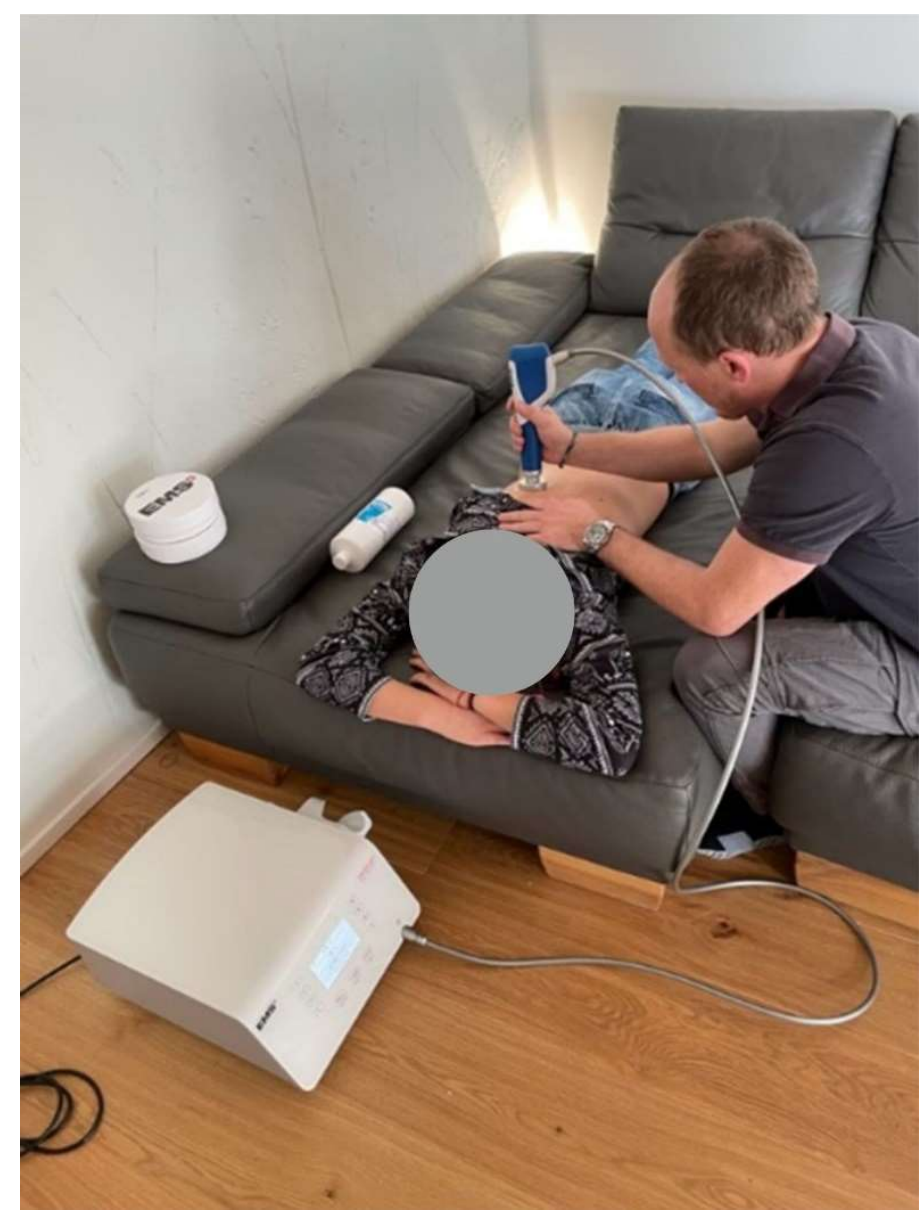

Figure 1. Treatment of a patient with COVID-19 using radial extracorporeal shock wave therapy applied over the intercostal muscles from dorsal. For illustration purposes the picture was taken without protective measures after the patient was cured and no longer positive for SARS-CoV-2.

\section{DISCUSSION}

To our knowledge this individual case report is the first one demonstrating that rESWT is able to achieve fast and effective relief of pain and pain-related breathing problems in therapyresistant respiratory distress caused by COVID- 19 .

There are limitations to this case report. Specifically, only a single patient was investigated and no control treatment was performed. However, it was not the aim of this case report to establish a clinical treatment. It goes without saying that both safety and efficacy of our novel approach must be confirmed in future studies, including well-designed randomized controlled trials on a larger number of patients.

\section{CONCLUSION}

Treatment of shortness of breath in conjunction with severe chest pain using rESWT may help many patients with COVID-19 or patients who still suffer from breathing problems weeks after having been infected with SARS-CoV2 , giving them back quality of life. 


\section{Acknowledgements}

The authors acknowledge the help of the patient and her parents in consenting to publish their case details.

\section{Declaration of conflicting interests}

P.S. declares no potential conflicts of interest with respect to the research, authorship and/or publication of this article. C.S. received (until June 30, 2020) research funding from Electro Medical Systems (Nyon, Switzerland) for his preclinical research at LMU Munich (unrestricted grant) and consulted (until December 31, 2017) for Electro Medical Systems. However, Electro Medical Systems had no any role in study design, data collection and analysis, interpretation of the data, decision to publish and writing the manuscript. No other potential conflicts of interest relevant to this article were reported.

\section{Ethical approval}

Our institutions do not require ethical approval for reporting individual cases.

\section{References}

1. Zhu J, Ji P, Pang J, Zhong Z, Li H, He C, Zhang J, Zhao C. Clinical characteristics of 3062 COVID-19 patients: A meta-analysis. J Med Virol 2020; 92(10): 1902-1914.

2. Oleynick C. Symptoms of pleurisy as the initial presentation of COVID-19. Am J Case Rep 2020; 21:e925775.

3. Császár NB, Angstman NB, Milz S, Sprecher CM, Kobel P, Farhat M, Furia JP, Schmitz C. Radial shock wave devices generate cavitation. PLoS One 2015; 10(10): e0140541.

4. Hochstrasser T, Frank HG, Schmitz C. Dose-dependent and cell type-specific cell death and proliferation following in vitro exposure to radial extracorporeal shock waves. Sci Rep 2016; 6: 30637.

5. Schmitz C, Császár NB, Milz S, Schieker M, Maffulli N, Rompe JD,

\section{Informed consent}

Written informed consent was obtained from the patient and the patient's parents (having parental authority) for the patient's anonymized information to be published in this article.

\section{Funding}

The authors received no financial support for the research, authorship and/or publication of this article.

\section{Availability of data and materials}

All datasets used and analyzed are presented in this paper.

\section{Authors' contributions}

P.S.: treatment, data collection, initial draft of manuscript. C.S.: guide and final editing of manuscript. All authors read and approved the final manuscript.

\section{ORCID iD}

Christoph Schmitz: https://orcid.org/0000-0002-4065-1241

Furia JP. Efficacy and safety of extracorporeal shock wave therapy for orthopedic conditions: a systematic review on studies listed in the PEDro database. Br Med Bull 2015; 116(1): 115-138.

6. Reilly JM, Bluman E, Tenforde AS. Effect of shockwave treatment for management of upper and lower extremity musculoskeletal conditions: a narrative review. PM\&R 2018; 10(12): 1385-1403.

7. Maier M, Averbeck B, Milz S, Refior HJ, Schmitz C. Substance P and prostaglandin E2 release after shock wave application to the rabbit femur. Clin Orthop Relat Res 2003; (406): 237-245.

8. Vidal X, Martí-Fàbregas J, Canet $\mathrm{O}$, Roqué M, Morral A, Tur M, Schmitz C, Sitjà-Rabert M. Efficacy of radial extracorporeal shock wave therapy compared with botulinum toxin type A injection in treatment of lower extremity spasticity in subjects with cerebral palsy: A randomized, controlled, cross-over study. J Rehabil Med 2020; 52(6): jrm00076. 